\title{
ON MULTIPLICATIVE REPRESENTATIONS OF INTEGERS
}

\author{
Dedicated to George Szekeres on his 65th birthday \\ P. ERDÖS AND A. SZEMERÉDI \\ (Received 1 December 1974) \\ Communicated by Jennifer Seberry Wallis
}

Abstract

Let $1 \leqq a_{1}<\cdots<a_{k} \leqq x ; b_{1}<\cdots<b_{1} \leqq x$. Assume that the number of solutions of $a_{1} b_{1}=m$ is less than $c$. The authors prove that then

$$
k l<\frac{c_{1} x^{2}}{\log x}(\log \log x)^{\prime(c)} .
$$

They also give a simple proof of Szemerédi's theorem: If the products $a_{i} b_{j}$ are all distinct then

$$
k l<\frac{c_{2} x^{2}}{\log x} \quad \text { (i.e. } f(1)=0 \text { ). }
$$

They conjecture that (2) holds for $c_{2}=1+\varepsilon$ if $x>x_{0}(\varepsilon)$. Several other unsolved problems are stated.

Let $a_{1}<\cdots<a_{k} \leqq x$ be a sequence of integers for which the products $a_{i} a_{j}$ are all distinct. Erdös proved that

$$
\pi(x)+c_{2} x^{3 / 4} /(\log x)^{3 / 2}<\max k<\pi(x)+c_{1} x^{3 / 4} /(\log x)^{3 / 2} .
$$

Perhaps there is an absolute constant $c$ so that

$$
\max k=\pi(x)+c x^{3 / 4} /(\log x)^{3 / 2}+\sigma\left(\frac{x^{3 / 4}}{(\log x)^{3 / 2}}\right)
$$

but we can not prove (1). (c, $c_{1}, \cdots$ denote absolute constants not necessarily the same.)

Erdös (1964a) also proved that if $a_{1}<\cdots<a_{k} \leqq x$ is such that the number of solutions of $a_{i} a_{i}=t$ is less than $2^{l}+1$ then

$$
\max k=(1+\sigma(1)) \frac{n(\log \log n)^{l-1}}{(l-1) ! \log n} .
$$

In fact (2) holds if the number of solutions is $\leqq 2^{i-1}+1$.

Let $a_{1}<\cdots$, denote by $g(n)$ the number of solutions of $n=a_{i} a_{j}$. (2) easily 
implies that if $g(n)>0$ for all $n$ then $\lim \sup _{n=\infty} g(n)=\infty$. It is curious to remark that the additive analogoues of this result present great difficulties. An old problem of Erdös and Turán states: Denote by $f(n)$ the number of solutions of $n=a_{i}+a_{j}$. Then if $f(n)>0$ then $\limsup _{n=x} f(n)=\infty$. The proof or disproof of this conjecture seems to present surprising difficulties and Erdös offered 300 dollars for a proof or disproof of this conjecture.

Raikov proved that if $a_{1}<\cdots$ is such that $g(n)>0$ for all $n$ then

$$
\limsup _{x=x} A(x) \frac{(\log x)^{1 / 2}}{x}>0
$$

where $\boldsymbol{A}(x)=\Sigma_{a_{i}<x} 1$. P. Erdös asked: Is there a sequence $a_{1}<\cdots$ for which $g(n)>0$ and $A(x)<c x / \log x$ for infinitely many $x$. Wirsing (1957) answered this question affirmatively, in fact he showed that $g(n)>0$ for all $n>n_{0}$ implies $A(x)>x / \log x(1+\varepsilon)$ for some $\varepsilon>0$ and that this result is best possible; that is, for every $\varepsilon>0$ there is a sequence $a_{1}<\cdots$ satisfying $g(n)>0$ for all $n>n_{0}$ and $A(x)<x / \log x(1+\varepsilon)$ for infinitely many $x$.

Let $1 \leqq a_{1}<\cdots<a_{k} \leqq x, 1 \leqq b_{1}<\cdots<b_{1} \leqq x$. Assume that there are at least $c x$ distinct integers not exceeding $x$ of the form $a_{i} b_{j}$. Then $\max (A(x), B(x))>x^{\frac{1}{2}+\varepsilon}$ and if the number of distinct $a_{i} b_{j}$ 's is $x+\sigma(x)$ then $\max (A(x), B(x))>x^{1-\varepsilon}$ for every $\varepsilon>0$. We do not discuss the proofs here which are not difficult.

It might be worth while to investigate that if $g(n)>0$ and $A(x)<c x / \log x$ holds for infinitely many $x$ is it then true that $A(x)>c x$ for infinitely many $x$, or if this would not be true, how fast must $A(x)$ increase for a suitable infinite sequence $x_{i} \rightarrow \infty$.

One more question in this direction: Let $a_{1}<\cdots<a_{k} \leqq x$ be a sequence of integers for which the products $\Pi_{i=1}^{k} a_{i}^{\varepsilon_{i}}, \varepsilon_{i}=0$ or 1 are all distinct. Erdös (1966) proved $k<\pi(x)+c x^{1 / 2} / \log x$ and probably

$$
\max k=\pi(x)+\pi\left(x^{1 / 2}\right)+\sigma\left(\frac{x^{1 / 2}}{\log x}\right) .
$$

In fact, perhaps the following more precise statement can be made: Let $1 \leqq u_{1}<\cdots<u_{k}$ be a sequence of integers for which all the sums $\sum_{i=1}^{k} \varepsilon_{i} u_{i}, \varepsilon_{i}=0$ or 1 are all distinct. Put $\min u_{k}=\alpha_{k}$. Erdös-and Pósa observed that

$$
\max k \geqq \sum_{k=1}^{\infty} \pi\left(x^{1 / \alpha_{k}}\right)
$$

Ind there could be equality in (3). A very old problem of Erdös asks: Is it true hat $\alpha_{k}>2^{k-c}$ for every $k$ where $c$ is an absolute constant?

Let $1 \leqq a_{1}<\cdots<a_{k} \leqq x ; 1 \leqq b_{1}<\cdots<b_{t} \leqq x$ be two sequences on inte- 
gers. Assume that all the products $a_{i} b_{;}, 1 \leqq i \leqq k ; 1 \leqq j \leqq l$ are distinct. Erdös conjectured and Szemerédi proved that then [Szemerédi (to appear)]

$$
k l<\frac{c x^{2}}{\log x} .
$$

First of all we give a simpler proof of (4), which nevertheless uses many of the ideas of the original proof. We conjecture that in fact

$$
k l \leqq(1+\sigma(1)) \frac{x^{2}}{\log x} .
$$

It is easy to see that (5) if true is best possible. To see this, let the $a$ 's be the primes in $(x / t, x)$ and the $b$ 's are the integers not exceeding $x$ all whose prime factors are $\leqq x / t$. Clearly the products $a_{i} b_{j}$ are all distinct and the prime number theorem implies $k l \geqq(1+\sigma)) x^{2} / \log x$ if $t=t_{x} \rightarrow \infty$ but $t / x^{\varepsilon} \rightarrow 0$ for every $\varepsilon>0$. In fact by choosing $t=\log x(1+\sigma(1))$ we maximize $k l$ and we then get sequences $a_{1}<\cdots<a_{k} ; b_{1}<\cdots<b_{1}$ with the products $a_{i} b_{j}$ all distinct and

$$
k l>\frac{x^{2}}{\log x}-\frac{x^{2} \log \log x}{(\log x)^{2}}+\sigma\left(\frac{x^{2} \log \log x}{(\log x)^{2}}\right) .
$$

It would be of interest to see if (6) can be improved. Conceivably it is best possible, but we have no evidence for it.

In this paper we prove the following theorem. To every $c$ there is an $f(c)$ so that if $1 \leqq a_{1}<\cdots<a_{k} \leqq x ; 1 \leqq b_{1}<\cdots<b_{l} \leqq x$ are such that $g(n) \leqq c$ for all $n$ then

$$
k l<\frac{c_{1} x^{2}}{\log x}(\log \log x)^{f(c)} .
$$

(7) is best possible apart from the value of $f(c)$. The proof is not entirely trivial and we only outline it. Let $r>1$ be given. The sequence $B$ consists of all the squarefree integers $b$ satisfying $x / 2<b<x$, and $v(b) \leqq r(v(b)$ is the number of prime factors of $b$ ). The sequence $A$ consists of all the integers $a<x$ which do not have two divisors $d_{1}<d_{2}<2 d_{1}, v\left(d_{1}\right) \leqq r, v\left(d_{2}\right) \leqq r$.

It is not difficult to show that

$$
A(x)>c_{1} x, B(x)>c_{2} x(\log \log x)^{r} / \log x
$$

and the number of solutions of $a_{i} b_{j}=n$ is less than $c_{r}$ where $c_{r}$ depends only on $r$. We do not discuss the details.

We further outline the proof of the following two theorems:

1. Assume $A(x)>c_{1} x, B(x)>c_{2} x$. Then

$$
\max _{n \leqq x^{2}} g(n)>(\log x)^{c_{3}}
$$


Again apart from the value of $c_{3}$ this is best possible. (To see this, let the $a$ 's and $b$ 's have $\leqq \log \log n$ prime factors). Finally assume $A \cup B$ is the set of all integers and $A(x)>c x, B(x)>c x$. Here

$$
\max _{n \leqq x^{2}} g(n)>(\log x)^{c^{\log \log x}}
$$

and apart from the value of $c_{4}$ this is best possible. To see this, let the $a$ 's have $\leqq \log \log n$ prime factors and the $b$ 's have $>\log \log n$ prime factors. Perhaps (9) holds for every $c_{4}<1-\varepsilon$. The above example shows that it can not hold for $c_{4}>1+\varepsilon$.

Now we are ready to prove (4). In other words we prove the following.

THEOREM 1. Let $1 \leqq a_{1}<\cdots<a_{k} \leqq x ; 1 \leqq b_{1}<\cdots<b_{1} \leqq x$ be two sequences of integers. Assume that the products $a_{i} b_{j}$ are all distinct. Then for some absolute constant $c$

$$
k l<\frac{c x^{2}}{\log x} .
$$

Denote by $A$ respectively $B$ the sequences $\left\{a_{1}, \cdots, a_{k}\right\}$ and $\left\{b_{1}, \cdots, b_{1}\right\}$. $A(y)$ will denote the number of terms of $A$ not exceeding $y$. A prime $p$ is associated with $A$ if there are at least $k / 100 p \log p$ multiplies of $p$ in $A$, similarly $p$ is associated with $B$ if there are at least $l /(100 p \log p)$ multiples of $p$ in $B$. Let $p_{1}<p_{2}<\cdots$ be the primes which are not associated with $A-$ omit all the $a$ 's which are multiples of any of the $p$ 's. Thus we obtain the new sequence $A_{1}$ having $k_{1}$ terms. Repeat the same process and also apply it to $B$ with the primes not associated with $B$. Since $\Sigma_{p}(1 /(100 p \log p))<\frac{1}{2}$, eventually we obtain a sequence $U=\left\{u_{1}<\cdots<u_{\lambda_{1}}\right\}, U \subset A, \lambda_{1}>k / 2$ and $V=\left\{v_{1}<\cdots<v_{\lambda_{2}}\right\}, V \subset B$, $\lambda_{2}>l / 2$ with the property that if $p \mid u_{i}$ then $p$ is associated with $U$ and if $p \mid v_{i}$ then $p$ is associated with $V$. To prove our theorem it clearly suffices to show

$$
\lambda_{1} \lambda_{2}<c_{1} x^{2} / \log x
$$

Let now $t\left(2^{t}<x^{1 / 2}\right)$ be the greatest integer for which there are more than $2^{t / 2} p$ 's in $\left(2^{t}, 2^{t+1}\right)$ which are associated with both $U$ and $V$. Denote these primes by $p_{1}, \cdots, p_{s}$.

$$
2^{t}<p_{1}<\cdots<p_{s}<2^{t+1}, \quad s>2^{t / 2} .
$$

Consider the set of all pairs of integers

$$
\left\{\frac{u_{i}}{p_{i}}, \frac{v_{j}}{p_{i}}\right\}, \quad 1 \leqq i \leqq s
$$

where $u_{j} \equiv 0\left(\bmod p_{i}\right), v_{j^{\prime}} \equiv 0\left(\bmod p_{i}\right)$. Since $p_{i}$ is associated with both $U$ and $V$ there are by (11) at least $\left(\lambda_{1}=U(x), \lambda_{2}=V(x)\right)$ 


$$
\frac{c \lambda_{1} \lambda_{2}}{10^{4}(t+1)^{2} 2^{2 t+2}}>\frac{c \lambda_{1} \lambda_{2}}{t^{2} 2^{3 t / 2}}
$$

pairs (12).

Now observe that the pairs (12) are unique. If

$$
\alpha=\frac{u_{j_{1}}}{p_{i_{1}}}=\frac{u_{j_{2}}}{p_{j_{2}}} \quad \text { and } \quad \beta=\frac{v_{j i}}{p_{i_{1}}}=\frac{v_{j 2}}{p_{j_{2}}}
$$

then $u_{j_{1}} v_{j_{2}}=u_{j_{2}} v_{j_{1}}=\alpha \beta p_{i_{1}} p_{i_{2}}$ which contradicts our assumptions.

Now we estimate the number of pairs (12) from above. Denote by $2^{t+1}<P_{1}<\cdots<x$ the primes associated with both $U$ and $V$. By the maximality of $t$ there are at most $2^{l / 2}$ primes $P$ in the interval $\left(2^{l}, 2^{l+1}\right)$ for every $l>t$. Thus trivially

$$
\sum_{i} \frac{1}{P_{i}}<8
$$

Denote by $q_{1}<\cdots$ the primes in $\left(2^{t+1}, x\right)$ not associated with $U$ and by $r_{1}<\cdots$ the primes in $\left(2^{t+1}, x\right)$ not associated with $V$. Clearly the integers (12) satisfy

$$
\frac{u_{i}}{p_{i}}<\frac{x}{2^{\prime}}, \frac{v_{j^{\prime}}}{p_{i}}<\frac{x}{2^{\prime}} \text { and } \frac{u_{j}}{p_{i}} \not \equiv 0(\bmod q), \frac{v_{j^{\prime}}}{p_{i}} \equiv 0(\bmod r)
$$

for all the primes $q$ and $r$ defined above. By Brun's method we immediately obtain from (15) that the number of integers of the form $u_{j} / p_{i}$ is less than

$$
c_{1} \frac{x}{2^{\prime}} \pi\left(1-\frac{1}{q_{i}}\right)
$$

and the number of integers of the form $v_{i} / p_{i}$ is less than

$$
c_{2} \frac{x}{2^{t}} \pi\left(1-\frac{1}{r_{i}}\right)
$$

Thus, from (16) and (17) we obtain that the number of pairs (12) is less than

$$
c_{1} c_{2} \frac{x^{2}}{2^{2 t}} \pi\left(1-\frac{1}{q_{i}}\right) \pi\left(1-\frac{1}{r_{i}}\right) .
$$

From (14) and the theorem of Mertens we obtain

$$
\sum \frac{1}{q_{i}}+\sum \frac{1}{r_{i}}=\sum_{1} \frac{1}{p}-\sum \frac{1}{P_{i}}>\log \log x-\log t-c
$$

where in $\Sigma_{1}$ the summation is extended over all the primes in $\left(2^{t+1}, x\right)$. From (18) and (19) we obtain that the number of pairs (12) is less than

$$
c_{3} \frac{x^{2} t}{2^{2 t} \log x} \text {. }
$$


From (13), (20) and the uniqueness of the pairs (12) we thus obtain

$$
\frac{c \lambda_{1} \lambda_{2}}{t^{2} 2^{3 t / 2}}<\frac{c_{3} x^{2} t}{2^{2 t} \log x}
$$

or

$$
\lambda_{1} \lambda_{2}<c \frac{x^{2} t^{3}}{2^{t / 2} \log x}
$$

which proves (10) and completes the proof of Theorem 1.

Observe that if no $t$ exists for which there are many primes in $\left(2^{t}, 2^{t+1}\right)$ associated with both $U$ and $V$ the proof gives

$$
U(x) V(x)<c x^{2} / \log x
$$

if there is a large $t$ then in fact $U(x) V(x)=\sigma\left(x^{2} / \log x\right)$.

Now let us try to obtain $A(x) B(x)<(1+\sigma(1)) x^{2} / \log x$. One can formulate this as an extremal problem in number theory. Assume $1 \leqq a_{1}<\cdots<a_{k} \leqq x$; $1 \leqq b_{1}<\cdots<b_{1} \leqq x$ are such that the products $a_{i} b_{j}$ are all distinct. What is the maximum of $k l$ and which sequences realize this maximum. Perhaps the sequence defined in the introduction comes close but we have no evidence. One could try first of all to prove that the extremal sequence has the following structure: Split the primes into two classes $q_{i}$ and $r_{j}$. The $A$ 's are the integers composed of the $q$ 's and the $B$ 's are the integers composed of the $r$ 's. We have not been able to show this - the method which we use in proving Theorem 1 gives: We can assume that the extremal sequence has the following structure: The primes are split into three classes $\left\{q_{i}\right\},\left\{r_{i}\right\}\left\{s_{i}\right\} \Sigma 1 / s_{l}<C$ and all the $q$ 's are associated with $A$, all the $r$ 's with $B$ and the $s$ 's can be associated with both.

If we would succeed in eliminating the primes $s$ then to prove $A(x) B(x)<$ $(1+\sigma(1)) x^{2} / \log x$ we would need the following theorem on sieves which we can not prove but which perhaps can be attacked by the experts: Let $q_{1}<\cdots$; $r_{1}<\cdots$ be two disjoint sequences of primes $a_{1}<a_{2}<\cdots ; b_{1}<b_{2}<\cdots$ are the integers composed of the $q$ 's and $r$ 's respectively. Is it true that

$$
A(x) B(x) \leqq(1+\sigma(1)) \frac{x^{2}}{\log x} .
$$

As shown in the introduction, equality is possible in (21), but perhaps the only way to achieve equality in (21) is to have $\min \left(\Sigma 1 / q_{i}, \Sigma 1 / r_{i}\right)$ tend to 0 as $x \rightarrow \infty$.

Theorem 2. Let $A(x)>c_{1} x, B(x)>c_{2} x$. Then for some $n<x, g(n)>$ $\log x)^{\alpha}$.

Theorem 2 is an immediate consequence of an old theorem of Erdös (1960). 
The number of products of the form $a_{i} b_{j}$ is $>c_{1}^{2} x^{2}$ but there are fewer than $x^{2} /(\log x)^{\alpha}$ distinct integers of the form $k l, k<x, l<x$. This implies Theorem 2 .

It would be interesting to determine the best possible value of $\alpha, \alpha \leqq 1 / \log 2$ is easy to prove, and at present it is not clear to us how much this can be improved.

THEOREM 3. Let $A(x)>c x, B(x)>c x$ and assume that every $m<x$ is either in $A$ or $B$. Then for some $n<x$ and $x>x_{0}(\varepsilon)$,

$$
g(n)>(\log x)^{\left(\frac{1}{4}-\varepsilon\right) \log \log x}
$$

Denote by $I$ the interval ( $\eta$ is sufficiently small)

$$
\left(c^{(\log x) \eta}, c^{(\log x)^{1 / 2}}\right)
$$

and $p_{1}<\cdots<p_{s}$ be the primes in $I$. Put

$$
k=\left[\frac{1}{2}(\log x)^{1 / 2}\right], \quad l=\sum_{i=1}^{s} \frac{1}{p_{i}}=\left(\frac{1}{2}-\eta\right) \log \log x+0(1)
$$

Denote by $D$ the sequence $d_{1}<d_{2}<\cdots$ of integers not exceeding $x$ which have at least $k$ distinct prime factors in $I$. It is easy to see that

$$
D(x)>\frac{x}{2 \log x} l^{k-1} /(k-1) !
$$

The proof of (23) follows the method of Hardy and Ramanujan (1920) and will be suppressed.

Without loss of generality we can assume that at least $\frac{1}{2} D(x)$ of the $d$ 's are in $A$ (since $A \vee B$ contains all the integers not exceeding $x$ ).

It follows from Turán's method (1934) that all but $\sigma(x)$ integers not exceeding $x$ have $l+\sigma(l)$ distinct prime factors in $I$. Thus by. $B(x)>c x$ we can assume that at least $c x / 2$ of the $b$ 's have at least $t$ distinct prime factors in $I$ where $t=[(1-\varepsilon) l]$. Consider now all the integers

$$
a_{i} b_{j}, a_{i} \in D \cap A, V\left(b_{j}\right) \geqq t .
$$

By (24) the number of these products is greater than

$$
\frac{x^{2}}{(\log x)^{2}} l^{k-1} /(k-1) !
$$

It is not difficult to see that almost all of these products are squarefree and these then have at least $k+l$ prime factors in $I$. It is easy to see that the number of integers not exceeding $x$ which have at least $k+l$ distinct prime factors in $I$ is less than 


$$
x\left(\sum_{i=1}^{s} \frac{1}{p_{i}}\right)^{k+l-1} /(k+l-1) !=x l^{k+l-1} /(k+l-1) ! .
$$

From (25) and (26) we obtain that there is an $n$ for which the number of solutions of $n=a_{i} b_{j}$ is at least

$$
\frac{x}{(\log x)^{2}} l^{k-1} /(k-1) !\left(\frac{x l^{k+1-1}}{(k+l-1) !}\right)^{-1}>\frac{k^{l}}{l^{\prime}}>(\log x)^{\left(\frac{1}{4}-\varepsilon\right) \log \log x}
$$

which proves (21).

Perhaps (21) holds with $1-\epsilon$ instead of $\frac{1}{4}-\varepsilon$. To make the proof work, I would have to be the interval

$$
\left(c^{(\log x)^{\eta}}, c^{(\log x)^{1-\eta}}\right), \quad k=\left[(\log x)^{1-\eta}\right] .
$$

But then we could not prove (23), but we hope to return to this question.

Finally we prove

THEOREM 4. To every $c$ there is an $f(c)$ so that if $1 \leqq a_{1}<\cdots<a_{k} \leqq x$; $1 \leqq b_{1}<\cdots<b_{l} \leqq x$ are such that $g(n)<c$ then (7) holds.

For simplicity we only prove this for $c=4$. Assume that

$$
k l>\frac{x}{\log x}(\log \log x)^{\alpha}
$$

where $\alpha$ is sufficiently large. We are going to prove that (27) implies that there are integers $z, y$ and four primes $p_{1}^{(0)}, p_{1}^{(1)}, p_{2}^{(0)}, p_{2}^{(1)}$ so that for all choices of $\varepsilon_{i}=0$ or $1, i=1,2$,

$$
y \prod_{i=1}^{2} p_{i}^{\left(\varepsilon_{i}\right)} \in A, \quad z \cdot \prod_{i=1}^{2} p_{i}^{\left(\varepsilon_{i}\right)} \in B .
$$

(28) clearly implies that $g\left(z y p_{1}^{(0)} p_{1}^{(1)} p_{2}^{(0)} p_{2}^{(1)}\right) \geqq 4$, hence to prove Theorem 4 it suffices to prove (28). In view of the fact that we do not try to get best possible values of $\alpha$, the proof of this will in some respect be simpler than the proof of Theorem 1.

We say that the prime $p$ belongs to $A$ if there are at least $k /\left(p(\log \log p)^{2}\right)$ multiples of it in $A$. This is a slight modification of the definition in Theorem 1 (which as the attentive reader will later see is really needed here) but since $\Sigma 1 /\left(p(\log \log p)^{2}\right)$ converges, this makes no difference.

Let $t_{1}$ be the smallest integer satisfying $(\log x)^{c}<2^{i_{1}}<x^{1 / 2}$ (where $c$ is sufficiently large) for which there are more than $2^{t_{1}} /\left(t_{1}\left(\log t_{1}\right)^{2}\right)$ primes which belong to both $A$ and $B$. If no such interval exists, then Brun's sieve gives as in the proof of Theorem 1 that $k l<c x^{2} \log \log x / \log x$ which implies that in this case our theorem holds. 
Let now

$$
p_{1}, p_{2}, \cdots, p_{s}, \quad s>\frac{2^{t_{1}}}{t_{1}\left(\log t_{1}\right)^{2}}
$$

be the primes in $\left(2^{i_{1}}, 2^{t_{1}+1}\right)$ which belong to both $A$ and $B$. Denote by $A_{p_{i}}$ respectively $B_{p_{i}}$ the set of integers

$$
\left\{\frac{a_{j}}{p_{i}}\right\},\left\{\frac{b_{j^{\prime}}}{p_{i}}\right\}, \quad a_{i} \equiv 0\left(\bmod p_{i}\right), \quad b_{i^{\prime}} \equiv 0\left(\bmod p_{i}\right) .
$$

Let now $t_{2}^{(i)}$ be the smallest integer satisfying $(\log x)^{c}<2^{t_{2}^{(i)}}<\left(x / p_{i}\right)^{1 / 2}$ for which there are more than $2^{h^{(i)}} /\left(t_{2}^{(i)}\left(\log t_{2}^{(i)}\right)^{2}\right)$ primes $p_{i}^{(i)}$ in $\left(2^{h_{2}^{(i)}}, 2^{\xi_{2}^{(i)+1}}\right)$ which belong to both $A_{p_{i}}$ and $B_{p_{i}}$. If such a $t_{2}^{(i)}$ does not exist then every prime $q$ in $\left((\log x)^{c},\left(x / p_{i}\right)^{1 / 2}\right)$ belongs to at most one of the sequences $A_{p_{i}}, B_{p_{i}}$ (we neglected a set of primes the sum of whose reciprocals goes to 0 as $x \rightarrow \infty$ and which may belong to both $A$ and $B$ ). But then as in the proof of Theorem 1 we obtain by Brun's method

$$
\left|A_{p_{i}}\right|\left|B_{p_{i}}\right|<\frac{c x^{2} \log \log x}{p_{i}^{2} \log x} .
$$

Thus from (30) and the definition of $A_{p_{i}}, B_{p_{i}}$ we have

$$
k l=|A||B| \leqq\left|A_{p_{i}}\right|\left|B_{p_{i}}\right| p_{i}^{2}\left(\log \log p_{i}\right)^{4} \leqq \frac{c x^{2}(\log \log x)^{5}}{\log x}
$$

which again proves Theorem 4.

The number of possible for $t_{2}^{(i)}$ is at most $\log x$, thus there are at least

$$
\frac{2^{t_{1}}}{t_{1}\left(\log t_{1}\right)^{2} \log x}
$$

primes $p_{i}$ (in $\left(2^{t_{1}}, 2^{t_{1}+1}\right)$ ) which have the same $t_{2}$.

Let $p_{i}, 1 \leqq i \leqq s$ be the primes (28) and $q_{1}, \cdots, q_{r}$ the set of primes in $\left(2^{t_{2}}, 2^{t_{2}+1}\right)$. To every $p_{i}$ there are at least $2^{t_{2}} /\left(t_{2}\left(\log t^{2}\right)^{2}\right) p_{j}^{(i)}$ 's (which are $q$ 's) so that there are at least

$$
\begin{aligned}
& \frac{c k}{p_{i} p_{j}^{(i)}\left(\log \log p_{i}\right)^{2}\left(\log \log p_{i}^{(i)}\right)^{2}}>\frac{c k}{2^{i_{1}+t_{2}}\left(\log t_{1}\right)^{2}\left(\log t_{2}\right)^{2}} \\
& >\frac{c x}{2^{t_{1}+t_{2}}\left(\log t_{1}\right)^{2}\left(\log t_{2}\right)^{2} \log x} \quad\left(\text { since } k>\frac{x}{\log x}\right)
\end{aligned}
$$

integers $u<x / p_{i} p_{j}^{(i)}$ so that $u p_{i} p_{j}^{(i)} \in A$. Therefore by a simple computation there is an integer $U$ to which there are at least

$$
\frac{2^{i_{1}+h_{2}}}{(\log x)^{3}}
$$


products $p_{i} p_{i}^{(i)}$ for which $U p_{i} p_{j}^{(i)} \in A$. Henceforth we only consider these pairs $p_{i} p_{i}^{(i)}$ which belong to $U$. To each of these pairs there are at least

$$
\frac{c x}{\log x 2^{t_{1}+t_{2}}\left(\log t_{1}\right)^{2}\left(\log t_{2}\right)^{2}}
$$

integers $v<x / p_{i} p_{i}^{(i)}$ so that $v p_{i} p_{j}^{(i)} \in B$. Thus again by a simple averaging process there is a $V$ so that there are at least

$$
\frac{2^{t_{1}+t_{2}}}{(\log x)^{5}}
$$

pairs $p \cdot q$ for which $U p q \in A, V p q \in B$.

Now we use following simple lemma on graphs. Let $G$ be a bipartite graph of $L_{1}$ white and $L_{2}$ black vertices and more than

$$
L_{1}^{1 / 2} L_{2}
$$

edges $\left(L_{1}<L_{2}\right)$. Then the graph contains a rectangle. Since $2^{t_{1}}>(\log x)^{100}$, $2^{12}>(\log x)^{100}$, the lemma applies and the rectangle gives the information which we require.

For $c=2^{k}$ the proof is similar. We have to apply our procedure $k$ times and have to use the theorem on $k$-tuples in (1964b).

\section{References}

P. Erdös (1969), 'On some applications of graph theory to number theoretic problem', Publ. Ramanujan Inst. 1, 131-136.

P. Erdös (1969a), 'On the multiplicative representation of integers,' Israel J. Math. 2, $251-261$.

P. Erdös (1966), 'Extremal Problems in Number Theory', (written in Hungarian), Mat. Lapok 17, $135-155$.

P. Erdös (1960), 'On an asymptotic formula in number theory', Leningrad Univ. 15, 41-49.

P. Erdös (1964b), 'On extremal problems of graphs and generalized graphs, Israel J. Math. 2, $183-190$.

G. H. Hardy and S. Ramanujan (1920), Quarterly J. Math. 48, 76-92; see also Collected papers of Srinivasa Ramanujan.

E. Szemerédi, 'On a problem of P. Erdös', Journal of Number Theory (to appear).

P. Turán (1934), 'On a theorem of Hardy and Ramanujan', J. London Math. Soc. 9, $274-276$.

E. Wirsing (1957), 'Über die Dichte multiplikativer Basen', Archiv der Math. 8, 11-15.

Department of Computer Science, Stanford University, California, U.S.A. 